\title{
Tromboflebite séptica de seio cavernoso com formação de pseudoaneurisma em
}

\section{criança de sete anos}

\author{
Septic cavernous sinus thrombophlebitis with formation of pseudoaneurysm in a 7-year-old child \\ Tromboflebitis séptica del seno cavernoso con formación de pseudoaneurisma en un niño de siete \\ años
}

Recebido: 01/03/2021 | Revisado: 09/03/2021 | Aceito: 13/03/2021 | Publicado: 20/03/2021

\author{
Júlia Vieira Ferreira Côrtes \\ ORCID: https://orcid.org/0000-0002-2760-7473 \\ Universidade José do Rosário Vellano, Brasil \\ E-mail: guiliavi@ hotmail.com \\ Larissa Terra Freire \\ ORCID: https://orcid.org/0000-0002-3646-8276 \\ Universidade José do Rosário Vellano, Brasil \\ E-mail: lariterra@outlook.com \\ Sabrina Silva Rodrigues de Oliveira \\ ORCID: https://orcid.org/0000-0002-4326-5100 \\ Universidade José do Rosário Vellano, Brasil \\ E-mail: sabrinasroliveira@yahoo.com \\ Gérsika Bitencourt Santos \\ ORCID: https://orcid.org/0000-0003-0849-2786 \\ Universidade José do Rosário Vellano, Brasil \\ E-mail: gersika.santos@unifenas.br \\ Débora Mônica Costa Vieira \\ ORCID: https://orcid.org/0000-0002-5280-7661 \\ Universidade José do Rosário Vellano, Brasil \\ E-mail: debmonica@hotmail.com \\ Renato Ortolani Marcondes de Castro \\ ORCID: https://orcid.org/0000-0002-1456-0442 \\ Universidade José do Rosário Vellano, Brasil \\ E-mail: renato.castro@prof.unifenas.br
}

\begin{abstract}
Resumo
A trombose séptica do seio cavernoso é uma complicação rara de infecções envolvendo os seios paranasais e pode levar a formação de aneurismas carotídeo-cavernosos. O objetivo deste estudo foi descrever o caso clínico de um paciente com um pseudoaneurisma micótico na artéria carótida, após um quadro de sinusite esfenoidal que evoluiu para uma síndrome cavernosa. Paciente feminino, sete anos de idade, com histórico recente de sinusite esfenoidal, apresentou sinais de irritação meníngea, confusão mental e febre de forte intensidade. A avaliação da ressonância nuclear de crânio mostrou áreas sugestivas de infartos agudos secundários à vasculite por meningite e tromboflebite do seio cavernoso esquerdo, com formação de um pseudoaneurisma na artéria carótida interna. Evoluiu com ptose e paralisia completa do globo ocular à esquerda, associada a edema periorbitário à esquerda com turvação visual. Recebeu antibioticoterapia por aproximadamente cinco semanas e foi realizado procedimento endovascular com boa evolução clínica posterior. Portanto, este estudo destaca a importância de uma alta suspeição diagnóstica para instituir o tratamento precoce e aumentar a sobrevida, evitando a morbimortalidade infantil.
\end{abstract}

Palavras-chave: Seio cavernoso; Trombose dos seios intracranianos; Celulite orbitária; Meningite.

\begin{abstract}
Septic cavernous sinus thrombosis is a rare complication of infections involving paranasal sinuses and can lead to carotid cavernous aneurysm formation. Thus, the objective of this study was to describe the clinical case of a patient with internal carotid artery mycotic pseudoaneurysm secondary to a case of sphenoid sinusitis that evolved into a cavernous sinus syndrome. A seven-year-old female patient with a recent history of sphenoid sinusitis, showed signs of meningeal irritation, mental confusion and severe fever. Brain magnetic resonance imaging showed areas suggestive of acute infarctions secondary to vasculitis due to meningitis and thrombophlebitis of the left cavernous sinus, with the formation of an internal carotid artery pseudoaneurysm. The patient evolved with ptosis and complete paralysis of the left eye, associated with unilateral periorbital edema on the left, with visual turbidity. After antibiotic therapy for approximately five weeks, an endovascular procedure was performed, with a good clinical outcome. Therefore, this study highlights
\end{abstract}


the importance of a high diagnostic suspicion to institute early treatment and increase survival, avoiding infant morbidity and mortality.

Keywords: Cavernoussinus; Intracranial sinus thrombosis; Orbital cellulitis; Meningitis.

\begin{abstract}
Resumen
La trombosis séptica del seno cavernoso es una complicación rara de las infecciones que afectan a los senos paranasales y puede conducir a la formación de aneurismas carótido-cavernosos. Así, el objetivo de este estudio fue describir el caso clínico de un paciente con un pseudoaneurisma micótico en la arteria carótida, tras un cuadro de sinusitis esfenoidal que evolucionó a un síndrome cavernoso. Una paciente de siete años con antecedentes recientes de sinusitis esfenoidal mostró signos de irritación meníngea, confusión mental y fiebre intensa. La evaluación de la resonancia nuclear mostró áreas sugestivas de infartos agudos secundarios a vasculitis por meningitis y tromboflebitis del seno cavernoso izquierdo, con formación de un pseudoaneurisma en la arteria carótida interna. Evolucionó con ptosis y parálisis completa del globo ocular del lado izquierdo, asociado a edema periorbitario del lado izquierdo con turbidez visual. Recibió antibioterapia durante aproximadamente cinco semanas y posteriormente se realizó un procedimiento endovascular con buen resultado clínico. Por tanto, este estudio destaca la importancia de una alta sospecha diagnóstica para instaurar un tratamiento temprano y aumentar la supervivencia, evitando la morbimortalidad infantil.
\end{abstract}

Palabras clave: Seno cavernoso; Trombosis de dos convulsiones intracraneales; Órbita de la celulitis; Meningitis.

\title{
1. Introdução
}

A síndrome do seio cavernoso é qualquer processo de patologias envolvendo o seio carvernoso (Kuybu \& Dossani, 2020). Este é uma estrutura vital para a artéria carótida interna e para os pares cranianos III, IV, V e VI. Assim, qualquer processo infeccioso ou trombose nesta área pode ser seguida por um grupo de manifestações clínicas, incluindo ptose, oftalmoplegia, diplopia e parestesia ao redor da cavidade orbitária (Weerasinghe \& Lueck, 2016). As principais etiologias são: vasculares que incluem fístulas carotídeo-cavernosas, aneurismas carotídeo-cavernosos e trombose do seio cavernoso; tumores do seio cavernoso; causas infecciosas e inflamatórias (Kuybu \& Dossani, 2020).

As causas da trombose do seio cavernoso são assépticas, como traumas, ou infecciosas, como celulite orbitária, e o agente etiológico mais comum é o Staphylococcus aureus (Martel, 2017). A trombose séptica é uma complicação rara, mas grave, das infecções deste seio e costuma ser devido a processo supurativo ao nível da órbita, seios paranasais ou metade superior da face, tais como sinusite e pode levar a formação de aneurismas (Kuybu \& Dossani, 2020). Apesar de ser afecção incomum, sua sintomatologia clínica é bem conhecida e deve ser prontamente identificada, pois o diagnóstico tardio piora ainda mais o prognóstico desta doença (Martel, 2017).

Este estudo tem como objetivo, relatar um caso de síndrome cavernosa, desenvolvida devido a uma sinusite esfenoidal em uma criança de sete anos com complicações neurológicas graves e raras, ressaltando a importância do conhecimento clínico para um diagnóstico e tratamento precoce evitando a morbimortalidade infantil.

\section{Metodologia}

Trata-se de um estudo de caso de característica qualitativa e descritiva. De acordo com Pereira et al. (2018), pesquisas desta natureza caracterizam-se por elucidar um determinado assunto e estudá-lo minuciosamente. Foi feita a revisão do prontuário, compreensão da história clínica, análise de exames de imagens e laboratoriais, registro dos métodos diagnóstico a que a paciente foi submetida. Todo o estudo foi realizado com base nos princípios da ética e da bioética, aprovado pela Resolução n 466, de 12 de dezembro de 2012, do Conselho Nacional de Saúde. A paciente assinou o Livre e Termo de Consentimento Livre e Esclarecido, no qual foram esclarecidos os objetivos da pesquisa e que a privacidade, confidencialidade e anonimato são assegurados. O estudo foi aprovado pelo comitê de ética em pesquisa da UNIFENAS sob parecer $\mathrm{n}^{\circ} 2.702 .359$. A busca das referências foi realizada com base nas pesquisas SciELO e PubMed. 


\section{Resultados e Discussão}

Paciente, sexo feminino, sete anos de idade, apresentou-se acompanhada da mão, ao pronto socorro com história de cefaleia holocraniana de forte intensidade, vômitos, tosse, febre e prostração há três dias. Ao exame físico de admissão, encontrava-se em regular estado geral, febril, desidratada 2+/4+, hipocorada 1+/4+, anictérica e acianótica. O exame neurológico estava normal, sem sinais meníngeos ou focais. Foi encaminhada para observação hospitalar, realizando reidratação endovenosa, uso de analgésicos, além de ser feita uma tomografia computadorizada de crânio espessamento do seio esfenoidal. Nos exames laboratoriais visualizou-se plaquetopenia.

Em seu primeiro dia de internação hospitalar apresentava-se agitada, com cefaleia intensa, turvação ocular, vertigem, além de ter apresentado seis episódios de febre alta. Ao exame físico evoluiu para rigidez de nuca e edema periorbitário associado com paralisia do globo ocular esquerdo. Com melhora da plaquetopenia, foi realizada punção lombar para coleta do líquido cefalorraquidiano (LCR), sem alterações. Como conduta foi prescrito tratamento empírico com Ceftriaxona $100 \mathrm{mg} / \mathrm{kg} / \mathrm{dia}$ e Aciclovir $30 \mathrm{mg} / \mathrm{kg} / \mathrm{dia}$, por 14 dias.

No sexto dia apresentou ptose palpebral e hiperemia conjuntival do olho esquerdo. Com a avaliação oftalmológica e ressonância magnética de crânio, a hipótese diagnóstica foi de Celulite Orbitária com oftalmoplegia restritiva. Vale ressaltar que os pares cranianos e a força estavam preservados, com pontuação do exame neurológico 15 na escala de Glasgow. A nova punção lombar de LCR revelou hipoglicorraquia e crescimento de Staphiloccocus Aureus. Foi prescrito Vancomicina $40 \mathrm{mg} / \mathrm{kg} / \mathrm{dia}$ e Meropenem $120 \mathrm{mg} / \mathrm{kg} / \mathrm{dia}$.

Após 14 dias de antibioticoterapia, a paciente estava estável, porém não apresentou melhora significativa do quadro, com persistência dos episódios febris. Nesse dia evoluiu para alteração do VI nervo craniano à esquerda evidenciado pela paralisia do músculo reto lateral esquerdo. Com a tomografia indicando espessamento do seio cavernoso esquerdo, foi feita angiografia cerebral, a qual evidenciou áreas de trombose do seio cavernoso esquerdo, com formação de um pseudoaneurisma na artéria carótida interna. (Figura 1). Ademais, a alteração no VI nervo persistia e ocorreu também alteração do III nervo à esquerda com sinais de ptose, estrabismo divergente e midríase não fotorreagente.

Figura 1. Angiografia por subtração da artéria carótida interna esquerda, diagnosticando pseudoaneurisma em segmento cavernoso com preenchimento do seio. Evidencia-se boa opacificação distal, sem sinais de embolias ou fístulas.

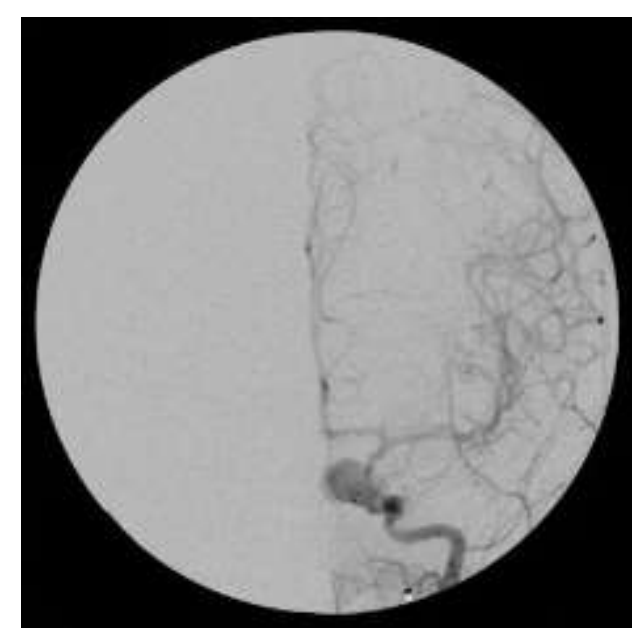

Fonte: Autores.

Com a avaliação de evidências radiológicas e clínicas, a hipótese diagnóstica foi síndrome cavernosa. Foi iniciada terapia empírica com Cefepima 100mg/kg/dia e Teicoplanina $20 \mathrm{mg} / \mathrm{kg} / \mathrm{dia}$ e solicitada vaga na Unidade de Terapia Intensiva até 
ser encaminhada para centro especializado onde realizou embolização vascular de aneurisma na artéria carótida interna esquerda. Vale ressaltar, a realização do teste de oclusão, o qual permitiu a concretização do procedimento endovascular (Figura 2).

FIgura 2. Controle angiográfico de teste de balão com oclusão da artéria carótida interna. Houve injeção em artéria vertebral esquerda, evidenciando boa opacificação da artéria cerebral média esquerda via artéria comunicante posterior.

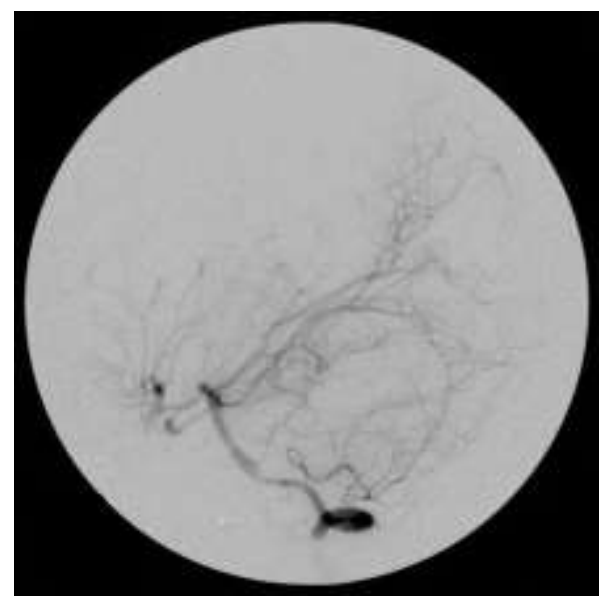

Fonte: Autores.

Com um cateter para acesso central, foi feita uma embolização na artéria carótida interna esquerda e, posteriormente, foi feita a oclusão dela com balão para ter um controle da embolização. Com outro cateter, foi feito o contraste na artéria carótida contra-lateral (Artéria Carótida Interna Direita) para que assim fosse possível visualizar se, mesmo com a oclusão da Carótica Interna Esquerda, teria a vascularização pelo polígono de Wiliis. Neste caso, foi observado que não houve a vascularização direta da Artéria cerebral Média, porém ocorreu vascularização da Artéria Vertebral, a qual irrigou as Artérias Comunicantes e, assim, o irrigamento da Artéria Comunicante Posterior fez a vascularização da Cerebral Média (Figura 3).

Figura 3. A. Controle final em perfil da artéria carótida comum, evidenciando oclusão da mesma pela técnica de trapping endovascular. B. Controle angiográfico final em towne da artéria carotida interna direita, evidenciando opacificação da artéria cerebral anterior via artéria comunicante anterior, não deixando ter ausência de fluxo cerebral. C. Controle angiográfico final, mostrando que há reperfusão pela artéria comunicante posterior.

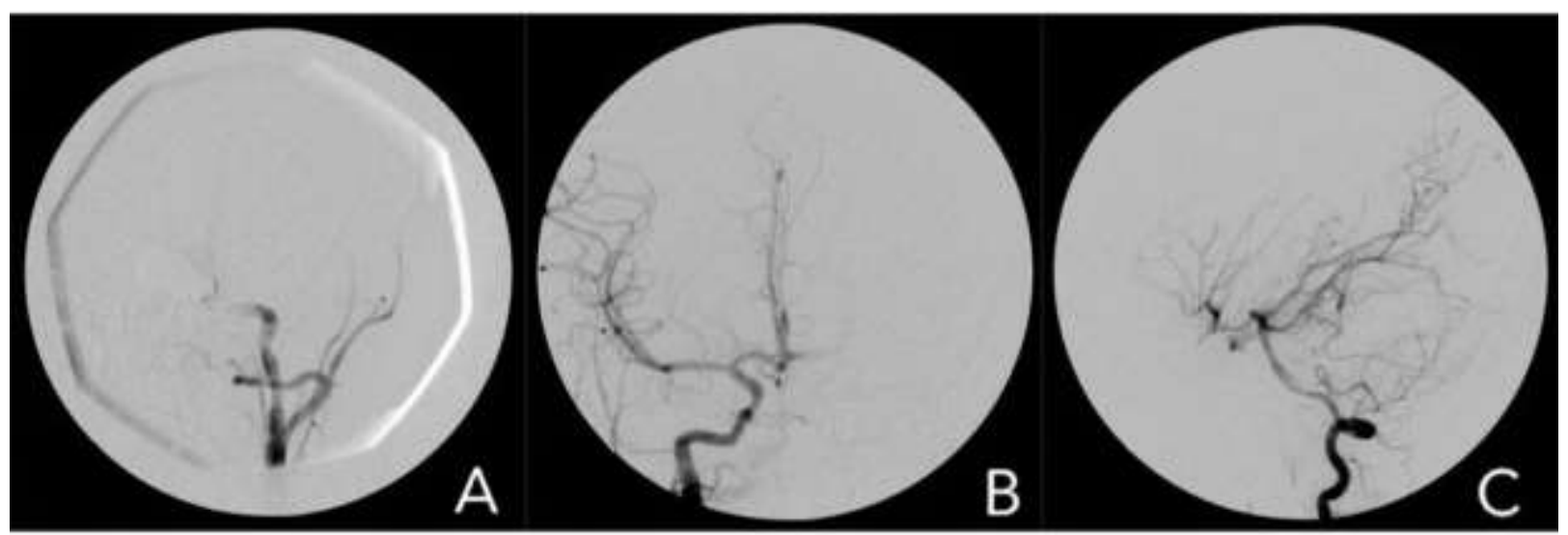

Fonte: Autores.

O procedimento foi realizado com sucesso e a paciente retornou para a unidade de terapia intensiva do hospital de origem. Após um dia foi para a enfermaria pediátrica mantendo antibiótico terapia com Cefepima 100mg/kg/dia e Teicoplanina 
$10 \mathrm{mg} / \mathrm{kg} /$ dia por mais 21 dias e acrescido Fluconazol 12mg/kg/dia por 21 dias. Ao final da terapêutica permaneceu afebril, com exame físico dentro da normalidade. Dessa forma, recebeu alta hospitalar com melhora do quadro e discreta ptose a esquerda que se manteve.

Destaca-se como um procedimento relevante diante desse caso, a embolização pela técnica de Trapping, que consiste no fechamento do vaso com oclusão, já que manteve a circulação via polígono de willis, através da vascularização das artérias vertebral, comunicantes e cerebral média.

Por razões anatômicas, os distúrbios que envolvem o seio cavernoso podem levar ao aparecimento de um conjunto de sinais e sintomas, designados por Síndrome do Seio Cavernoso. Estes podem ir desde a paralisia de nervos cranianos específicos até às alterações de sensibilidade da face (Costa et al, 2019). A importância deste relato de caso está na raridade de uma sinusite progredir a alterações neurológicas graves, ressaltando a necessidade deste estudo descritivo, no qual foi feita a revisão do prontuário, compreensão da história clínica, análise de exames de imagens e laboratoriais e registro dos métodos diagnóstico a que a paciente foi submetida.

A sinusite esfenoidal é um quadro clínico inespecífico e de difícil caracterização (Maia et al, 2018). As suas complicações mais frequentes são as infecções orbitárias como celulite, seguidas pelas intracranianas tais como meningites, abscessos intracerebrais e, menos frequentemente, trombose do seio cavernoso, a qual possui causas sépticas e assépticas (Maia et al, 2018). Isso se deve a fatores anatômicos, como, por exemplo, a abundância de veias diploicas e a proximidade com os seios paranasais (Dutta \& Ghatak, 2013). Este caso chama a atenção pelo fato de a paciente ter evoluído de um quadro de sinusite esfenoidal para uma celulite orbitária, concordando com a literatura acerca da anatomia da face facilitar a propagação de infecções. Posteriormente a infecção atingiu as meninges e o seio cavernoso, sendo as complicações mais graves de uma sinusite segundo a literatura.

A sintomatologia varia de acordo com o seio da face afetado e o principal sinal de alerta para complicações é a cefaleia, concomitante ao caso, visto que, a paciente apresentava cefaléia holocraniana de forte intensidade, devido à pressão intracraniana elevada que pode ocorrer como resultado de uma grande trombose de seio venoso (Idiculla et al, 2020).

Após a colonização da nasofaringe devido à sinusite esfenoidal, se inicia a fisiopatogenia da meningite bacteriana, que é um processo inflamatório que envolve as membranas cerebrais pia-máter e aracnoide e o líquido cefalorraquidiano, acometidas pelo agente etiológico mais comum, o Staphylococcus aureus. As manifestações clínicas variam de acordo com a idade podendo apresentar febre, hipotermia, letargia, vômitos, diarreia, dificuldade respiratória, bradicardia e sinais de irritação meníngea (Teixeira et al, 2018).

Toda essa inflamação atinge, através da anatomia, o seio cavernoso, visto que ele se estende pela fissura orbital superior e é delimitado pelas lâminas da meninge dura-máter no cérebro. Qualquer processo de doenças envolvendo esse seio dural, leva a Síndrome do Seio Cavernoso. As causas mais comuns da síndrome do seio cavernoso são as inflamatórias, seguidas dos aneurismas carotídeo-cavernosos, fístula carotídeo-cavernosa e, menos frequentemente, a tromboflebite (Kuybu \& Dossani, 2020).

A Trombose do Seio Cavernoso, complicação intracraniana, pode resultar de qualquer infecção do tecido drenado pelo seio cavernoso. A disseminação da infecção do seio esfenoidal prossegue principalmente pelas veias aferentes até o seio cavernoso não valvulado e resulta em trombose fulminante. Os microrganismos podem se espalhar diretamente de um seio esfenoidal infectado para o seio cavernoso através de veias comunicantes, via osteomielite do osso diplóide interveniente ou rompendo a mucosa na presença de defeitos ósseos. Conseqüentemente, essa infecção primária pode ter um prognóstico pior, levando, por exemplo, a um aneurisma micótico (Komatsu et al, 2013).

$\mathrm{O}$ aneurisma micótico é definido como uma falsa dilatação vascular causada que gera necrose e enfraquecimento da parede vascular. Esse tipo de aneurisma representa apenas 2-5\% de todos os aneurismas intracranianos, sendo, portanto, uma 
entidade considerada rara. Os aneurismas infecciosos podem se desenvolver por disseminação hematogênica de êmbolos sépticos ou, mais raramente, por contiguidade de uma infecção extravascular, como nas sinusites (Allen et al, 2013). Um fato interessante no presente caso é justamente a paciente ter um aneurisma micótico, tendo como etiologia, uma sinusite bacteriana.

Pacientes com trombose do seio cavernoso geralmente se queixam de febre, cefaleia, inchaço e dor periorbital e alterações na visão. Os sintomas podem estar presentes no início ou progredir subaguda ao longo de dias. Geralmente, começa com um olho e depois progride para outro. Os sintomas menos comuns podem incluir rigidez no pescoço, dormência facial, confusão, convulsões, sintomas de acidente vascular cerebral ou coma (Plewa et al, 2020). O principal sintoma que acomete esses pacientes é a oftalmoplegia, que pode persistir por semanas se não for tratada corretamente. A dor periorbital pode se estender às áreas retroorbital, temporal e frontal (Cantón-De-Seoane et al, 2020).

Os achados oculares são quase universais (90\%) (Plewa et al, 2020) e neste relato foram semelhando aos estudos anteriores. Isso inclui edema periorbital, eritema da pálpebra, quemose, ptose, proptose, movimento ocular restrito ou doloroso e, menos comumente, papiledema, hemorragia retiniana, diminuição da acuidade visual, fotofobia, reflexo pupilar diminuído e conjuntiva pulsante (Plewa et al, 2020).

À medida que a doença progride, os nervos cranianos podem ficar comprometidos e isso se manifesta como movimentos extra-oculares restritos, reflexos pupilares anormais e, em casos mais raros, até perda de visão (Geng et al, 2020). Mais uma vez, o relato está de acordo com os estudos, pois evoluiu com alteração do VI e III par craniano, ilustrado por paralisia do musculo reto lateral, ptose e midríase não fotorreagente.

O diagnóstico de Síndrome do seio cavernoso é desafiador devido a uma grande variedade de etiologias possíveis. Os estudos da órbita, sela e região paraselar desempenham um papel importante no diagnóstico (Henderson \& Miller, 2017). Sendo que a angiografia cerebral foi considerada o procedimento diagnóstico primário usado para o diagnóstico de anomalias venosas intracranianas (Idiculla et al, 2020).

Vários estudos têm demonstrado que o tratamento pode ser feito através da oclusão da artéria carótida. A maioria dos centros atualmente realiza a oclusão pela via endovascular, que quase sempre leva à completa trombose e consecutiva retração do aneurisma. Para se avaliar se o paciente tolerará a terapêutica oclusiva da carótida, realiza-se o teste de oclusão com balão (TOB), que consiste na insuflação temporária de um balão não destacável no interior da artéria carótida interna e monitoração do estado neurológico do paciente por um período de 10-30 minutos. A monitoração angiográfica associada à clínica durante o teste de oclusão tem-se mostrado um método confiável, de fácil realização, e não acrescenta custos ao procedimento (Ponte, et al 2006). A paciente em questão, ao final da embolização vascular do aneurisma na artéria carótida interna esquerda e terapêutica antibiótica, progrediu para exame físico dentro da normalidade e assim, adquiriu alta hospitalar.

Para concluir este relato, fica evidente que a clínica foi de suma importância para o diagnóstico do quadro, pois os aneurisma de carótida interna se manifestam geralmente por meio de sintomas compressivos aos nervos cranianos (Morita et al, 2011). Ademais a partir da suspeita da presença de uma lesão vascular, o "padrão ouro" para sua confirmação é a arteriografia cerebral, além de outros métodos como a angiotomografia e a angioressonância, as quais vêm sendo usados como alternativas menos invasivas para o diagnóstico e seguimento dessas lesões (Pierot et al, 2013). Acerca do tratamento escolhido, a maioria dos pacientes sintomáticos experimenta a melhora clínica ou mesmo cura dos déficits de nervos cranianos induzidos por aneurismas intracavernosos gigantes após o seu tratamento, seja por oclusão da Artéria Carótida Interna, ou mesmo por embolização endovascular. No entanto, o processo de melhora é dependente de diversos fatores como a duração prévia dos sintomas antes do tratamento, do início agudo ou gradual dos sintomas, do grau de disfunção dos Nervos Cranianos (Rooij et al, 2012). Por fim, o sucesso deste caso deve-se a intervenção precoce e escolha adequada do procedimento, evitando graves morbidades para a vida da paciente e evitando a mortalidade infantil. 


\section{Conclusão}

Este caso clínico ilustra a ocorrência de Síndrome Cavernosa, uma doença rara, que teve como etiologia uma sinusite esfenoidal com evolução para celulite orbitária, meningite bacteriana e trombose de seio craniano. Dessa forma, fica evidente a necessidade de um diagnóstico precoce, observando sinais de alertas de complicações de sinusites, para iniciar o quanto antes o tratamento preconizado e evitar a morbimortalidade infantil.

O presente relato esclarece que após o diagnóstico, o paciente foi tratado da maneira correta e permaneceu com mínimas sequelas, como ptose à esquerda. Dito isso, o objetivo foi alcançado, pois o intuito é de mostrar que quanto mais rápido a análise do quadro e a instituição do tratamento precoce melhor será o prognóstico da paciente. Foi visto, também, que o tratamento consiste na oclusão do aneurisma e da artéria carótida interna, após realização de teste de oclusão. Existem melhoras após realização do procedimento devido a redução da pressão dentro do aneurisma, porém algumas possíveis complicações seriam o surgimento de déficits pela redução de fluxo sanguíneo, ou o surgimento de aneurismas em outras localizações em razão do aumento compensatório do fluxo cerebral nas artérias do polígono de Willis.

Atualmente, vê-se pouca discussão sobre esse assunto e seu desfecho clínico, dado a sua relevância e gravidade. Sendo assim, torna-se necessário um estudo maior sobre o conteúdo, colaborando com mais relatos de casos, artigos e outros, fazendo com que a doença e suas sequelas se tornem mais conhecidas e por conseqüência, tratadas rapidamente. Aliado a essa proposta, aprofundar nos estudos relacionados a técnicas endovasculares, como embolização endossacular do aneurisma, oclusão do vaso portador, ou mesmo pela colocação de "stents" é fundamental, pois o desfecho deste caso se deve a presença de um serviço especializado de neurocirurgia e neuroradiologia onde foi realizado um procedimento complexo e invasivo que possibilitou a melhora da paciente.

\section{Referências}

Allen, L. M., Fowler, A. M., Walker, C., Derdeyn, C. P., Nguyen, B. V., Hasso, A. N., et al. (2013). Retrospective review of cerebral mycotic aneurysms in 26 patients: focus on treatment in strongly immunocompromised patients with a brief literature review. AJNR Am J Neuroradiol, 34, .823-827. https://doi.org/10.3174/ajnr.A3302

Cantón,-De-Seoane, J., et al. (2020). Síndrome de Tolosa-Hunt: una causa infrecuente de oftalmoplejía. Rev Esp Casos Clin Med Intern (RECCMI). 5(3), .120122. https://doi.org/10.32818/reccmi.a5n3a7

Costa, A. I., Lima, A., Azevedo, C., Quintas-Neves, M., Rodrigues, B., \& Dias, L. (2019). Pseudotumor inflamatório do seio cavernoso, síndrome de TolosaHunt e paralisia facial: Qual é a relação? Revista Portuguesa de Otorrinolaringologia e Cirurgia de Cabeça e Pescoço,57(2), 63-68. https://doi.org/10.34631/sporl.784

Dutta, M., \& Ghatak, S. (2013). Acute exacerbation of chronic rhinosinusitis (AECRS) with orbital complications in an atrophic rhinitis patient: a mere coincidence? J ClinDiagn Res, 7(12), .2973. https://doi.org/10.7860/JCDR/2013/6461.3814

Geng, B., et al. (2020). Septic cavernous sinus thrombosis-Case series and review of the literature. Clinical Neurology And Neurosurgery, $197, .106092$. http://dx.doi.org/10.1016/j.clineuro.2020.106092.

Henderson, A. D., \& Miller, N. R. (2017). Carotid-cavernous fistula: current concepts in aetiology, investigation, and management. Eye, 32(2), .164172. http://dx.doi.org/10.1038/eye.2017.240.

Idiculla, P. S, et al. (2020). Cerebral Venous Thrombosis: a comprehensive review. European Neurology. 83 (4), .369-379. https://doi.org/10.1159/000509802

Komatsu, H. et al. (2013). Cavernous sinus thrombosis caused by contralateral sphenoid sinusitis: a case report. Head \& Face Medicine, 9(1), .1-5. http://dx.doi.org/10.1186/1746-160x-9-9.

Kuybu, O., \& Dossani, R. H. (2020). CavernousSinusSyndromes. StatPearls. https://www.ncbi.nlm.nih.gov/books/NBK532976/

Maia, L. P., et al. (2018). Trombose de seio cavernoso e aneurisma micótico como complicações de rinossinusite aguda. Arquivos de Asma, Alergia e Imunologia, 2(2), .290-294. http://aaai-asbai.org.br/detalhe_artigo.asp?id=882

Martel, A. (2017). Septic thrombosis of cavernous sinus extended to the ipsilateral internal jugular vein and transversal sinus with favorable outcome: clinical and radiological features of a lemierre syndrome. Orbit, 37(2), .94-96. https://doi.org/10.1080/01676830.2017.1383458

Morita, K., Sorimachi, T., Ito, Y., Nishino, K., Jimbo, Y., Kumagai, T., \& Fujii, Y. (2011). Intra-aneurysmal coil embolization for large or giant carotid artery aneurysms in the cavernous sinus. Neurol Med Chir (Tokyo), 51(11) .762-766. http://dx.doi.org/10.2176/nmc.51.762. 
Research, Society and Development, v. 10, n. 3, e41010313443, 2021

(CC BY 4.0) | ISSN 2525-3409 | DOI: http://dx.doi.org/10.33448/rsd-v10i3.13443

Pereira, A. S. et al. (2018). Metodologia da pesquisa científica. UFSM. https://repositorio.ufsm.br/bitstream/handle/1/15824/Lic_Computacao_MetodologiaPesquisa-Cientifica.pdf?sequence $=1$.

Pierot, L., Portefaix, C., Rodriguez-Régent, C., Gallas, S., Meder, J. F., \& Oenheim, C. (2013). Role of MRA in the detection of intracranial aneurysm in the acute phase of subarachnoid hemorrhage. J Neuroradiol, 40(3) 204-10. http://dx.doi.org/10.1016/j.neurad.2013.03.004.

Plewa, M. C., et al. (2020). Cavernous Sinus Thrombosis. StatPearls. https://www.ncbi.nlm.nih.gov/books/NBK448177/

Ponte, F., et al. (2006). Aneurisma gigante do segmento intracavernoso da carótida interna associado a doença renal policística autossômica dominante: relato de caso. Arquivos de Neuro-Psiquiatria, 64 (3), 881-884. http://dx.doi.org/10.1590/S0004-282X2006000500034

Teixeira, A. B., et al. (2018). Bacterialmeningitis: anupdate. Revista Brasileira de Análises Clínicas, 50(4), 327-9. http://dx.doi.org/10.21877/24483877.201800725

Van Rooij, W. J. (2012). Endovascular treatment of cavernous sinus aneurysms. AJNR Am J Neuroradiol, 33(2), 323-326. http://dx.doi.org/10.3174/ajnr.a2759.

Weerasinghe, D., \& Lueck, C. J. (2016). Septic Cavernous Sinus Thrombosis: case report and review of the literature. Neuro-Ophthalmology, 40(6), .263-276. http://dx.doi.org/10.1080/01658107.2016.1230138. 